\title{
Retrograde Placement of Ring-Bolster Retained Tubes Facilitates Gastrostomy to Gastrojejunostomy Revision in Children with Esophageal Pathology and Microgastria
}

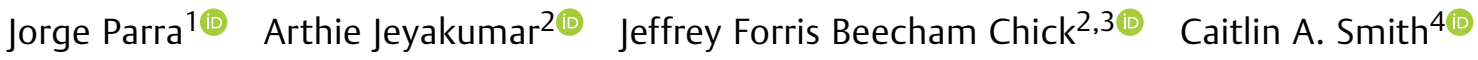 \\ Frederic J. Bertino 20 Eric J. Monroe 2,30
}

${ }^{1}$ College of Osteopathic Medicine, Pacific Northwest University of Health Sciences, Yakima, Washington, United States

2 Division of Interventional Radiology, Department of Radiology, University of Washington, Seattle, Washington, United States

${ }^{3}$ Division of Interventional Radiology, Department of Radiology, Seattle Children's Hospital, Seattle, Washington, United States

${ }^{4}$ Department of Surgery, University of Washington and Seattle Children's Hospital, Seattle, Washington, United States

\author{
Address for correspondence Eric J. Monroe, MD, Division of \\ Interventional Radiology, Department of Radiology, Seattle \\ Children's Hospital, 4800 Sand Point Way NE, Seattle, WA 98105 , \\ United States (e-mail: eric.j.monroe.md@gmail.com).
}

J Clin Interv Radiol ISVIR 2022;6:207-211.
Abstract
Keywords
- dysphagia
- nutrition
- gastrostomy
- gastrojejunostomy
- enteric access
- esophageal atresia
- tracheoesophageal fistula

Techniques for gastrostomy revision to gastrojejunostomy within the pediatric population, who have failed initial tube placement due to obstructive pathologies, have not been well established. Novel techniques are required to improve outcomes for these patients, and these techniques must be evaluated for both viability and safety. This article describes a technique utilized for gastrostomy to gastrojejunostomy revision and the outcomes for three pediatric patients.

\section{Introduction}

Gastrostomy tubes allow for safe, long-term delivery of enteral nutrition in those unable to maintain adequate nutrition orally. In infants, pathologies that may necessitate gastrostomy tube placement include congenital anomalies, malnutrition secondary to neuromuscular disorders, intractable food aversions, and alimentary dysfunctions. Although temporary enteral access may be obtained via a nasogastric or nasojejunal feeding tube, these tubes are prone to occlusion, malposition, and dislodgement. Therefore, a more durable option should be considered if required for $>8$ to 12 weeks.

In the pediatric population, indications for placement of a gastrostomy include enteral feeding, decompression of the gastrointestinal tract, and diversion of intestinal contents to article published online December 22, 2021
DOI https://doi.org/ 10.1055/s-0041-1740574. ISSN 2457-0214.
(C) 2021. Indian Society of Vascular and Interventional Radiology. All rights reserved.

This is an open access article published by Thieme under the terms of the Creative Commons Attribution-NonDerivative-NonCommercial-License, permitting copying and reproduction so long as the original work is given appropriate credit. Contents may not be used for commercial purposes, or adapted, remixed, transformed or built upon. (https://creativecommons.org/ licenses/by-nc-nd/4.0/)

Thieme Medical and Scientific Publishers Pvt. Ltd., A-12, 2nd Floor, Sector 2, Noida-201301 UP, India 
promote healing of intestinal fistulas. ${ }^{1}$ Fluoroscopic-guided placement of gastrostomy tubes provides an effective and safe alternative to endoscopic and surgical techniques. A meta-analysis by Wollman et al comparing fluoroscopicguided, endoscopic, and surgical-placed gastrostomy demonstrated fewer major complications using fluoroscopy (5.9\%) compared with endoscopy (9.4\%). ${ }^{2}$ Although surgical placement was $100 \%$ technically successful, complication rates were higher than fluoroscopic- or endoscopic-placed tubes $(19.9 \% ; p<0.001){ }^{2,3}$ Fluoroscopic-guided access techniques include antegrade, retrograde, and combination ("push-pull") methods.

Antegrade techniques entail passing the gastrostomy through the mouth, esophagus, and into the stomach. When performed by interventional radiology, this requires percutaneous access to the gastric lumen subsequently brought through the mouth by either orogastric snare capture and withdrawal or retrograde catheter and wire cannulation of the esophagus. ${ }^{4}$ Following oropharyngeal wire capture, the wire is exchanged for a snare. A ring-bolster retained gastrostomy is secured and withdrawn into final position from the percutaneous access. Coaxial placement of a 6- or 8-French feeding tube into the proximal small bowel completes a modular gastrojejunostomy.

Retrograde techniques require gastric insufflation, percutaneous suture anchor gastropexy, percutaneous needle and wire access to the stomach followed by tract dilation, and placement of a balloon- or pigtail-retained gastrostomy. ${ }^{3}$ Combination techniques utilize antegrade access to create an opening and retrograde techniques for gastrostomy placement.

Congenital gastrointestinal anomalies in pediatric patients pose unique challenges in establishing long-term nutritional access. Conditions such as tracheoesophageal fistula and esophageal atresia are associated with microgastria. Severe underdevelopment of the stomach has been shown to cause gastrostomy failure due to insufficient capacity resulting in gastric outlet obstruction. ${ }^{5}$ These patients also have significant reflux that often necessitates postpyloric feeding to minimize symptoms from reflux and improve weight gain. This can be a complex task in patients with recent esophageal anastomosis after repair of esophageal atresia.

Gastrostomy failure in these patients necessitates revision to gastrojejunostomy with intermittent or continuous gastric venting. Techniques for revision in this population are not well-established and are further complicated by difficulties obtaining the proper angle for gastrojejunostomy placement via the traditional entry points of preexisting gastrostomy. In patients with microgastria, the lower profile of ring-bolster retained gastrostomy tubes may be a preferred option. If related to esophageal pathology, however, traditional antegrade placement techniques are contraindicated due to the presence of a surgical anastomosis.

The objective of this article is to report a technique for gastrostomy to gastrojejunostomy revision with retrograde placement of a ring-bolster retained gastrostomy component in patients with microgastria and esophageal pathology precluding standard techniques.

\section{Materials and Methods}

\section{Patient Selection}

This study complied with the Health Insurance Portability and Accountability Act. Institutional review board approval was not required for this type of study. Pediatric patients undergoing gastrostomy to gastrojejunostomy revision using the technique described were included in the study. Between January 2016 and January 2018, three patients underwent enteric access revision as described. Technical success, complications, clinical success, and follow-up were recorded.

\section{Technique}

All procedures were performed by an attending pediatric interventional radiologist under general anesthesia. Existing gastrostomy tubes were removed over a 0.035-inch Amplatz wire which was then negotiated into the small bowel or looped within the gastric fundus using a 4-French angled catheter. The gastrostomy tracts were dilated over the wire with 20-French peel-away introducer sheath (Cook Medical, Bloomington, Indiana, United States). A 16-French ring-bolster retained gastrostomy tubes (CorFlo, Halyard, Alpharetta, Georgia, United States) was cut to approximately $20 \mathrm{~cm}$ length and loaded onto the percutaneous enteral access wire. Note that 19-gauge (3.2-French) angiocatheters were placed into the sidewall port for aspiration decompression of the gastrostomy ring bolsters. Peel-away sheaths were placed over the wires and over the gastrostomy tubes. Note that 6 -French vascular sheaths or JAKO fine suction tubes were placed over the wire and through the gastrostomy tubes for pushability. The decompressed ring bolsters were lubricated and withdrawn into the peelaway sheaths, followed by manual tapering of the distal sheaths. The constructs were advanced over the wire and through the gastrostomy tracts. The suction tube or vascular sheath and gastrostomy tubes were advanced as units beyond the peel-away sheaths and the peel-away sheaths were removed. The ring bolsters were reinflated using the angiocatheters and brought into apposition with the anterior gastric wall. Following contrast injection to confirm position and absence of extravasation, dual port feeding adaptors were placed (C.R. Bard, Tempe, Arizona, United States) followed by standard coaxial 8-French jejunal feeding tube placement. A benchtop demonstration of construct assembly is depicted in -Fig. 1 and a sample case is depicted in - Fig. 2 . A completed assembly hub is depicted in - Fig. 3. Standard stoma peristomal skin care including daily site wash with soap and soft cloth was initiated on postprocedure day 1 .

\section{Variables and Outcomes}

Technical success was defined as successful revision of gastrostomy to a ring-bolster retained gastrostomy tube and coaxial jejunal feeding tube. Clinical success was defined as successful initiation of postpyloric feeds. 

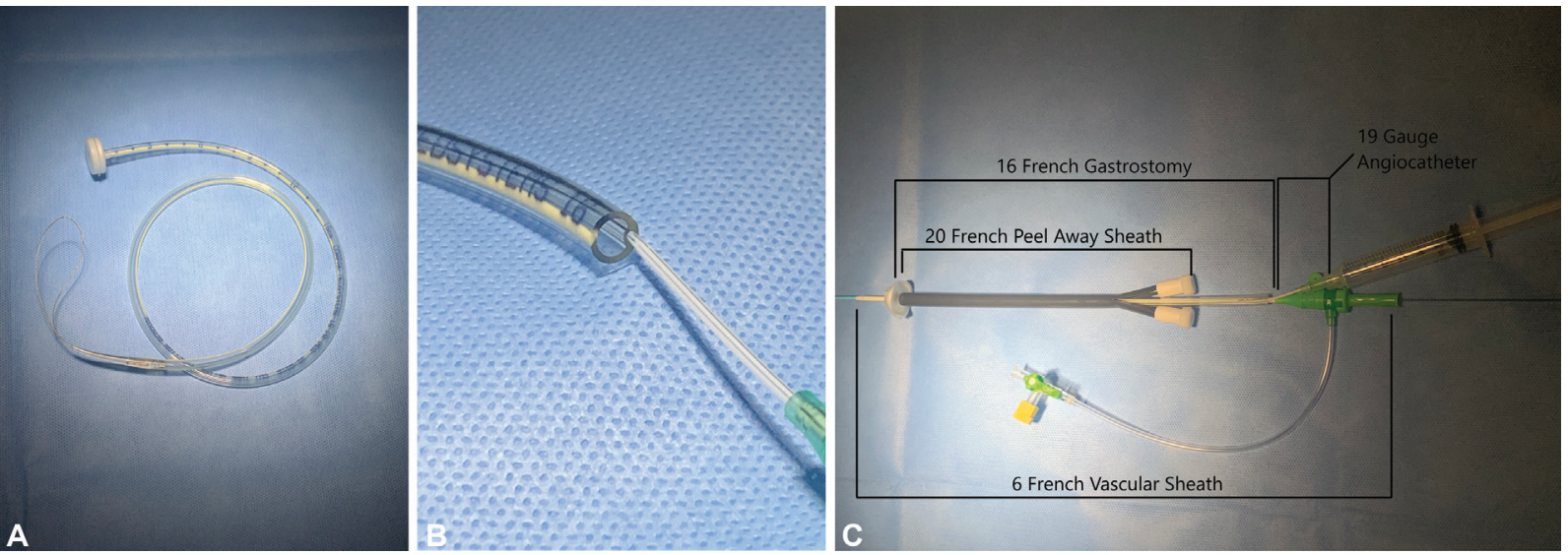

Fig. 1 Benchtop construct assembly. A 16-French ring-bolster retained gastrostomy as packaged (CorFlo, Halyard, Alpharetta, GA) (A). Transected gastrostomy tube with placement of an 18-gauge angiocatheter into the sidewall port for aspiration decompression of the gastrostomy ring bolster (B). Over-the-wire placement of the gastrostomy, 20-French peel-away introducer sheath (Cook Medical, Bloomington, IN) and inner 6-French vascular sheath prior to constraining the gastrostomy ring bolster within the peel-away (C).
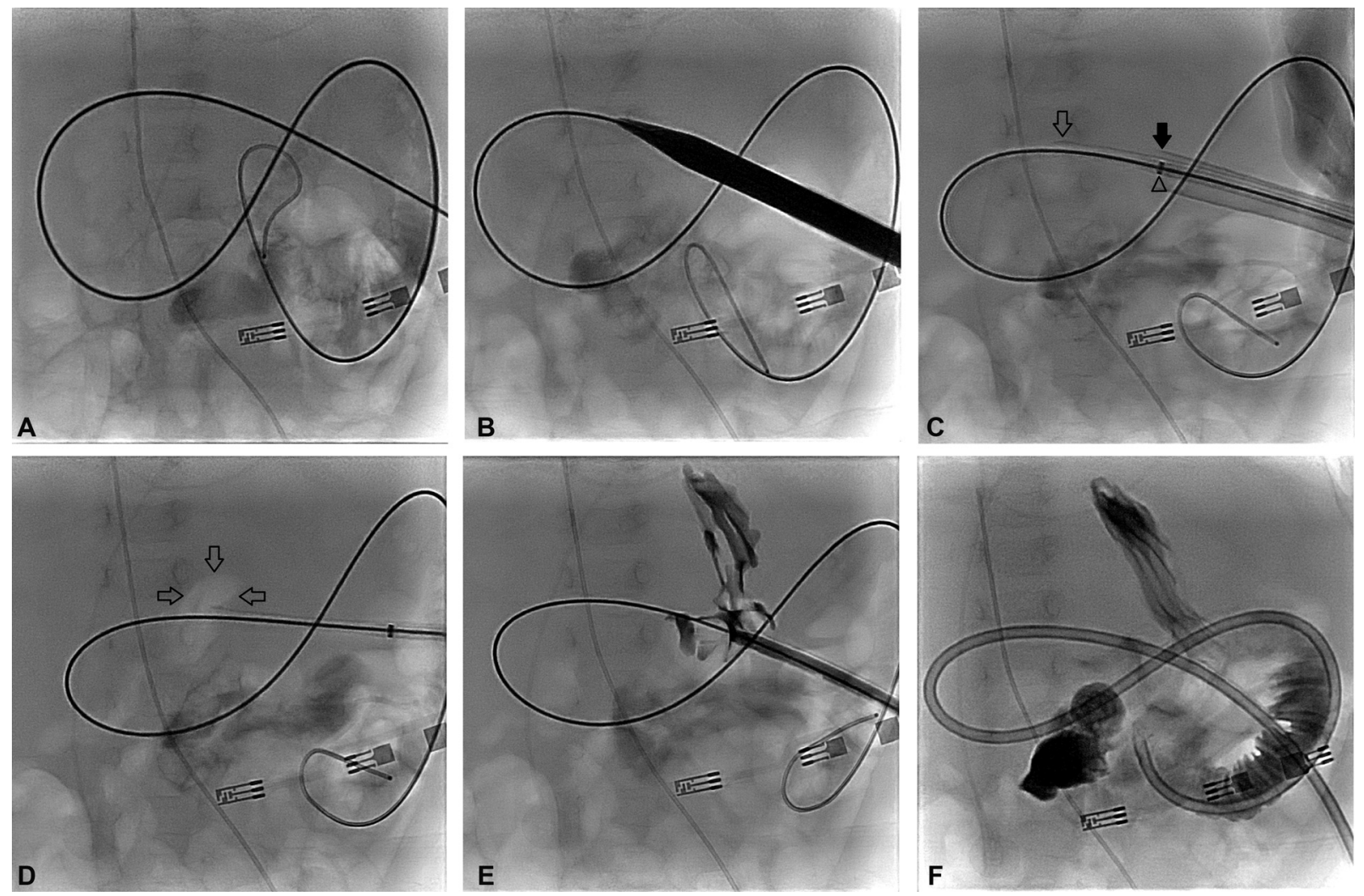

Fig. 2 Three-month-old male with CHARGE syndrome status postrepair of esophageal atresia, tracheoesophageal fistula ligation, and surgical gastrostomy tube placement, now requiring postpyloric feeds. Anteroposterior fluoroscopic image following removal of the surgical gastrostomy tube, jejunal catheterization, and 0.035-inch Amplatz wire placement (A). The tract is dilated with a 20-French dilator and peel-away sheath (B). A construct of 16-French CorFlo gastrostomy (open arrow), 6-French vascular sheath (open arrowhead), and constraining 20-French peel-away sheath (black arrow) are placed through the stoma and the CorFlo gastrostomy is subsequently advanced into the gastric lumen (C). The CorFlo gastrostomy ring bolster is reinflated (open arrows) and the peel-away sheath is removed (D). The gastrostomy disc is withdrawn into apposition with the anterior gastric wall and contrast injection confirms position (E). An 8-French feeding tube is placed coaxially into the jejunum, completing the gastrojejunostomy (F).

\section{Results}

Patient characteristics and results are summarized in - Table 1. Mean patient age and weight at time of revision were 5.7 months (range: 3-7 months) and $5.7 \mathrm{~kg}$ (range: 3.7-
$6.8 \mathrm{~kg}$ ), respectively. All patients had prior esophageal atresia repair. Initial gastrostomy placement was created with a 10French silicone Malecot catheters (Cook Medical LLC, Bloomington, United States). Two patients underwent surgical gastropexy at the time of gastrostomy. 


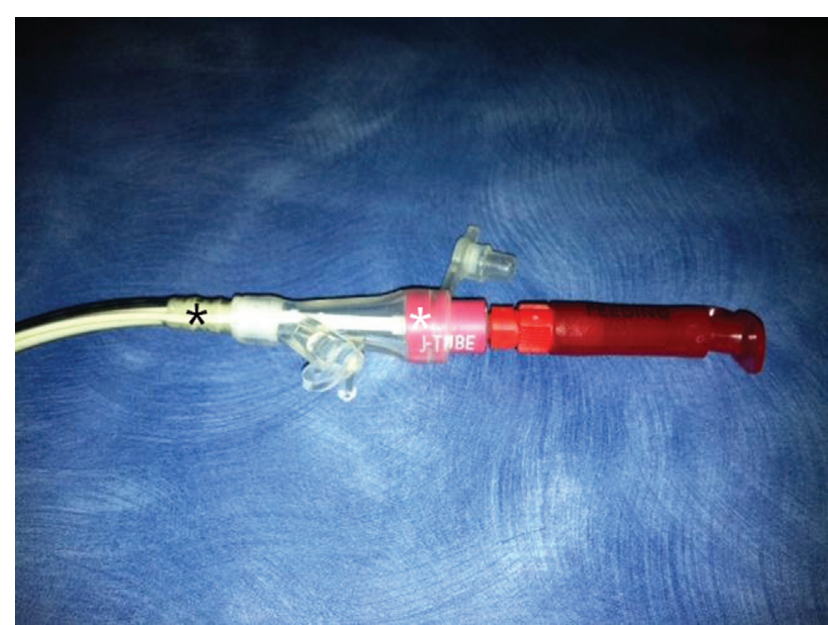

Fig. 3 Completed gastrojejunostomy assembly. Following placement of the CorFlo gastrostomy, a dual port feeding adaptor (C.R. Bard, Tempe, AZ) is placed at the cut end of the tube. Jejunal access is then achieved via coaxial 4-French catheter and 0.035-inch wire, followed by coaxial, over-the-wire placement of an 8-French jejunal feeding cut to length. Zip tie placement (not depicted) may be applied to both the adaptor entry point (black asterisk) and around the combination of the adaptor and feeding tube hub (white asterisk).

All revisions were technically successful. Mean fluoroscopy time and air kerma were 13.8 minutes (range: 8.818.7 minutes) and $21.8 \mathrm{mGy}$ (range: $12-27.4 \mathrm{mGy}$ ). There were no complications.

\section{Discussion}

Gastrostomy tubes are a common method of establishing long-term nutritional access in patients who are unable to feed orally. In the pediatric population, up to $6 \%$ require gastrostomy revision, most commonly due to prolapse of the stomach through the gastrostomy tract, migration of the gastrostomy site, excessive leakage, and chronic pain at the gastrostomy site. ${ }^{6}$ Establishing safer, minimally invasive methods of enteral access revision are necessary to optimize feeding and to troubleshoot dysfunction. Fluoroscopic gastrojejunostomy revision after surgical or endoscopic gastrostomy can be performed within weeks, provided gastropexy is used or in a delayed fashion ( $>8$ weeks) to allow tract maturation. The three cases described here demonstrate successful gastrojejunal (GJ) revision following failed initial gastric feeds due to reflux associated with esophageal pathology and gastric underdevelopment.

While no complications were encountered in these cases, risks associated with fluoroscopic GJ revision include possible disruption of surgical gastropexy or other iatrogenic gastric injury. Complications due to primary GJ placement include colocutaneous fistula, gastric outlet obstruction due to tube prolapse, and bezoar formation. ${ }^{7}$ Additionally, the time from gastrostomy placement to revision ranged from 3 to 8 months; however, earlier revision could result in higher complication rates due to less tract maturation and/or interval gastric growth. The described technique was prompted by a need for revision and concern that a balloon-retained GJ

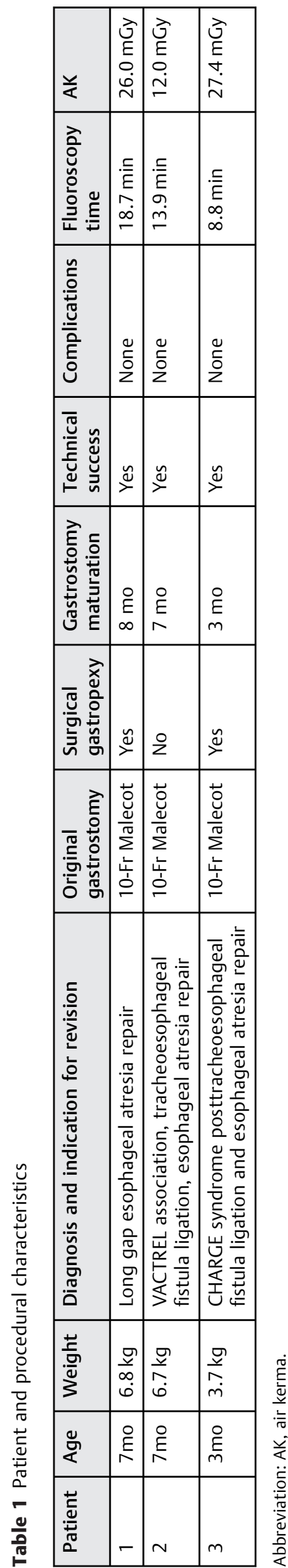


would lead to intolerance. In a series by Gill et al describing 34 patients undergoing de novo balloon-retained gastrojejunostomy, no instances of gastric outlet obstruction were observed despite generally small patient size (median weight $7.5 \mathrm{~kg}$, range: $2.9-31.6 \mathrm{~kg}){ }^{8}$ Although clinical indications varied, no patients in the Gill et al series had structural esophageal pathology with microgastria.

Primary limitations with regard to this case series include a small sample size; however, technical success in larger series is difficult to establish due to the infrequent presentation of patients with obstructive congenital pathologies. The methods described in this case series present a possible avenue for reestablishing safe, effective, and minimally invasive nutritional access in patients who have failed gastric feeds due to reflux or microgastria, or a combination of factors. While generalizability to different patient populations, and causes of failure, have not been established, the high rate of technical success and low rate of complications within this narrow subset of patients is promising.

\section{Conclusion}

Gastrostomy to gastrojejunostomy revision via retrograde placement of a ring-bolster retained gastrostomy component is a safe and feasible option for patients with intolerance of gastric feeds and esophageal pathology precluding standard techniques.

\section{Main Points}

- A ring-bolster retained gastrostomy tubes offer high retention forces with a low intraluminal gastric profile and allow coaxial placement of a 6- or 8-French feeding tube into the proximal small bowel to make a modular gastrojejunostomy.

- Antegrade techniques entail passing the gastrostomy through the mouth, esophagus, and into the stomach after percutaneous access to the gastric lumen and retrograde cannulation of the esophagus.

- Children with esophageal pathology may not be able to tolerate transesophageal placement of a ring-bolster retained gastrostomy tube, and associated microgastria introduces risk of gastric obstruction by comparatively larger profile and caliber of balloon-retained gastrojejunostomy tubes.

- The technique described in this note may be considered when clinical conditions necessitate retrograde placement of a ring-bolster retained gastrostomy tube.

\section{Authors' Contributions}

E.J.M. and J.F.B.C. equally contributed to the conception and design of the research; E.J.M. and J.F.B.C. contributed to the design of the research; E.J.M. and C.A.S. contributed to the acquisition and analysis of the data; E.J.M., J.F.B.C., F. J.B., and C.A.S. contributed to the interpretation of the data; and J.P. and A.J. drafted the manuscript. All authors critically revised the manuscript, agree to be fully accountable for ensuring the integrity and accuracy of the work, and read and approved the final manuscript.

\section{Financial Disclosures}

None declared.

Funding

This study was not supported by any funding.

All procedures performed in studies involving human participants were in accordance with the ethical standards of the institutional or national research committee and with the 1964 Helsinki Declaration and its later amendments or comparable ethical standards.

Conflict of interest

None declared.

\section{References}

1 Crowley JJ, Hogan MJ, Towbin RB, et al; Society of Interventional Radiology Standards of Practice Committee Society for Pediatric Radiology Interventional Radiology Committee. Quality improvement guidelines for pediatric gastrostomy and gastrojejunostomy tube placement. J Vasc Interv Radiol 2014;25(12):1983-1991

2 Wollman B, D'Agostino HB, Walus-Wigle JR, Easter DW, Beale A. Radiologic, endoscopic, and surgical gastrostomy: an institutional evaluation and meta-analysis of the literature. Radiology 1995; 197(03):699-704

3 Connolly B, Krishnamurthy G, Amaral J. Upper gastrointestinal access in children: techniques and outcomes. Tech Vasc Interv Radiol 2010;13(04):222-228

4 Reis J. Image-guided enteral access and interventions in children. Dig Dis Interv 2019;3(03):165-174

5 Chait PG, Weinberg J, Connolly BL, et al. Retrograde percutaneous gastrostomy and gastrojejunostomy in 505 children: a 4 1/2-year experience. Radiology 1996;201(03):691-695

6 Conlon SJ, Janik TA, Janik JS, Hendrickson RJ, Landholm AE. Gastrostomy revision: incidence and indications. J Pediatr Surg 2004;39(09):1390-1395

7 DeLegge $\mathrm{MH}$. Enteral access and associated complications. Gastroenterol Clin North Am 2018;47(01):23-37

8 Gill AE, Gallagher N, McElhanon BO, Painter AR, Gold BD, Hawkins $\mathrm{CM}$. Image-guided placement of percutaneous de novo low-profile gastrojejunostomy tubes in the pediatric population: a study of feasibility and efficacy. Pediatr Radiol 2018;48(06):882-888 